\title{
THE POWER SUBSTITUTION FOR RINGS OF COMPLEX AND REAL FUNCTIONS ON COMPACT METRIC SPACES
}

\author{
A. N. DRANISHNIKOV
}

(Communicated by James E. West)

\begin{abstract}
The weak power substitution property for rings of matrices over the ring of functions on a compact metric space $X$ is given in terms of cohomological dimension. A compactum with the ring of complex functions $C(X)$ having the following property is constructed: the units of $C(X)$ are not dense in $C(X)$ and they are dense among squares.
\end{abstract}

\section{INTRODUCTION}

The power substitution property for rings was introduced by Goodearl [1]. Then that property was applied to the following topological problem [2]:

Let $\eta, \xi_{1}$ and $\xi_{2}$ be complex or real vector bundles on a topological space $X$. Under what kind of conditions does the equality $\eta \oplus \xi_{1} \cong \eta \oplus \xi_{2}$ imply $m \xi_{1} \cong m \xi_{2}$ for some natural number $m \in \mathbf{N}$ ?

There is the natural bijection between complex (real) bundles on a compactum $X$ and projective modules over the ring of all complex (real) functions $C(X)$ which is defined by prescribing to a given vector bundle $\xi$ the group of its continuous sections $\Gamma(\xi)$. If the endomorphism ring of $\Gamma(\eta)$ satisfies the power substitution property, then the answer to the above question is affirmative.

A ring $R$ has the power substitution property if for any equality $a x+b y=1$ there exists a natural number $n$ such that $a 1_{n}+b M_{n}(R)$ contains a unit; here $M_{n}(R)$ denotes the ring of all $n \times n$ matrices over $R$ and $1_{n}$ denotes the identity matrix. For a commutative ring $R$ this property is the same as the following: whenever $a R+I=R$ for some ideal $I$ there exists a natural $n$ such that $a 1_{n}+M_{n}(I)$ contains a unit. For non-commutative rings the second property leads to the definition of the weak power substitution property. It's easy to check that the power substitution implies the weak power substitution. These definitions are taken from [3].

In this paper we show that the ring $M_{n}(C(X))$ has the weak power substitution property if and only if the compactum $X$ has cohomological dimension with respect to rationals less than or equal to three in the case of real-valued functions: $\operatorname{dim}_{\mathbb{Q}} X \leq 3$. In the case of complex-valued functions it's equivalent to $\operatorname{dim}_{\mathbb{Q}} X \leq 1$. This result is based on the main results of [3]. For $n=1,2$ it

Received by the editors November 30, 1993.

1991 Mathematics Subject Classification. Primary 55M10, 15A54; Secondary 16A54. 
was proved in [1]. The main result of this paper answers the following question by R. Camps: Does there exist an $m$-dimensional compactum $Y$ with $m \geq 2$ such that $C_{\mathbb{C}}(Y)$ has the power substitution property with fixed $n$ for all $a, b$ ? The answer is positive. In this case we shall call our ring $C_{\mathbb{C}}(Y)$ having the n-power subsitution property. We construct a compactum $Y$ of that sort with the property: the units are nowhere dense in $C=C_{\mathbb{C}}(Y)$ and they are dense in $C^{2}$, i.e. for every complex function $f$ the square $f^{2}$ can be approximated by units, i.e. functions missing zero in $\mathbb{C}$.

Still there is an open question to characterize compact metric spaces whose matrix rings of real (complex) functions have the power substitution ( $n$-power substitution) property. In particular does the weak power substitution property imply a power substitution for that kind of rings?

\section{WEAK POWER SUBSTITUTION}

Denote by $p_{m}: S O(n) \rightarrow S O(n m)$ the composition of taking the $m$-th power of matrices and the natural inclusion $S O(n) \rightarrow S(n m)$. Similarly, for the complex case denote its composition by $q_{m}: S U(n) \rightarrow S U(n m)$, and denote taking the $m$-th power for the circle by $(-)^{m}: S^{1} \rightarrow S^{1}$.

The following two propositions define the weak power substitution property for matrix rings over functional spaces in topological terms [3].

Proposition 1. For a compact space $X$ the matrix ring $M_{n}\left(C_{\mathbb{R}}(X)\right)$ over real functions has the weak power substitution property if and only if

(a) For every partial map $f: A \rightarrow S O(n)$ there is a natural number $m$ and an extension $\bar{f}: X \rightarrow S O(n m)$ of the map $p_{m} \circ f$.

Proposition 2. For a compact space $X$ the matrix ring $M_{n}\left(C_{\mathbb{C}}(X)\right)$ over complex functions has the weak power substitution property if and only if

(b) For every partial map $g: A \rightarrow S^{1}$ there is a number $m$ and an extension of the composition $g$ and $(-)^{m}$, and

(c) For every partial map $f: A \rightarrow S U(n)$ there is a number $m$ and an extension $\bar{f}: X \rightarrow S U(n m)$ of the composition $f$ and $q_{m}$.

By a partial map of a topological space $X$ we mean an arbitrary continuous map of a closed subset of $X$. Let $X$ and $M$ be topological spaces, and denote by $X \tau M$ the following extension property: for every partial map $f: A \rightarrow M$, there exists an extension $\bar{f}: X \rightarrow M$. The other notation for that is $M \in A E(X)$ which is to be read " $M$ is an absolute extensor for a space $X$ ". Let $N$ be a subspace of $M$. Then it's quite natural to denote by $X \tau(M, N)$ the above extension property where mappings $f$ with the ranges restricted to $N$ are considered. The next generalization of $X \tau(M, N)$ is to consider an arbitrary map $g: N \rightarrow M$ instead of an imbedding. Let us denote that extension property by $X \tau g$. For decent spaces $M$ and $N$ the last extension property $X \tau g$ is equivalent to the previous $X \tau\left(M_{g}, N\right)$ where $M_{g}$ denotes the mapping cylinder of the map $g$ and $N \subset M_{g}$ is the natural inclusion of the domain $N$ in the mapping cylinder.

By $K(G, n)$ we denote an Eilenberg-Mac Lane complex for a group $G$. I would like to recall that the extension property $X \tau K(G, n)$ means precisely that the cohomological dimension of $X$ is less than or equal to $n$ [4], [5]. So, 
that extension property can serve as a definition of cohomological dimension with respect to a group $G$. Notation is $\operatorname{dim}_{G} X$.

Let $S O_{(0)}, S O(n)_{(0)}, S U_{(0)}$ and $S U(n)_{(0)}$ be localizations at 0 of spaces $S O, S O(n), S U$ and $S U(n)$ [10]. Let $h_{k}$ denote a map generated by taking the $k$-th power in $S O$. Note that $S O_{(0)}$ is the direct limit of the sequence $M_{1} \rightarrow M_{2} \rightarrow M_{3} \rightarrow \cdots$ where the $k$-th space $M_{k}$ is the union of mapping cylinders of $h_{i}: S O \rightarrow S O$ with $i \leq k$, which is called a telescope of $\left(h_{i}\right.$ : $i \leq k)$, and the $k$-th bonding map is the inclusion of $M_{k}$ in $M_{k+1}$. The inclusion of $S O(n)$ in $S O$ defines the natural inclusion of $S O(n)_{(0)} \subset S O_{(0)}$ where $S O(n)_{(0)}$ is the direct limit of a corresponding subsequence of telescopes $N_{i}$. There is a similar construction for the pair $\left(S U_{(0)}, S U(n)_{(0)}\right)$. Note that the same construction works for the circle and the space $S_{(0)}^{1}$ is the EilenbergMac Lane space $K(\mathbb{Q}, 1)$.

Lemma 3. The conditions (a)-(c) of Propositions 1, 2 are equivalent respectively to:

$\left(\mathrm{a}^{\prime}\right) X \tau\left(S O_{(0)}, S O n_{(0)}\right)$,

(b') $\operatorname{dim}_{\mathbb{Q}} X \leq 1$,

(c') $X \tau\left(S U_{(0)}, S U(n)_{(0)}\right)$.

Proof. (a) $\Rightarrow\left(\mathrm{a}^{\prime}\right)$. Let $f: A \rightarrow S O(n)_{(0)}$ be a partial map. Since $A$ is compact, there is a number $i$ such that $f(A) \subset N_{i}$. Property (a) implies that there is a number $m$ such that the composition $p_{m} \circ \pi \circ f$ has an extension where $\pi: N_{i} \rightarrow S O(n)$ is the projection of the telescope onto the image of the last mapping. Note that $r \circ p_{m}=i_{n} \circ h_{i+m} \circ \cdots \circ h_{i+1}$ for some $r: S O(n m) \rightarrow S O$ and the natural inclusion $i_{n}: S O(n) \rightarrow S O$. The map $f$ is homotopic to $i_{n} \circ h_{i+m} \circ \cdots \circ h_{i+1} \circ \pi \circ f$, hence by the Homotopy Extension Theorem $f$ has an extension over $X$ to the map to $M_{i+m}$. Therefore $f$ has an extension to $S O_{(0)}$.

$\left(\mathbf{a}^{\prime}\right) \Rightarrow(\mathrm{a})$. Let $f: A \rightarrow S O(n)$ be a partial map. We may assume that $S O(n)$ is the first space in our telescope construction for $S O(n)_{(0)}$. Let $\bar{f}: X \rightarrow S O_{(0)}$ be an extension of $f$. Since $X$ is compact, there is a number $k$ such that $\bar{f}(X) \subset M_{k}$ and even more, it lies in some $k$-telescope $L_{k}$ of $S O(l)$ 's contained in $M_{k}$. If $l \leq k$, then (a) is checked with $m=k$. If $l \geq k$, we consider the projection on the $l$-level in our direct system and then (a) holds with $m=l$.

A compactness of $X$ easily implies the equivalence $(b) \Leftrightarrow\left(b^{\prime}\right)$.

The proof of $(c) \Leftrightarrow\left(c^{\prime}\right)$ is similar.

Lemma 4. For finite dimensional compacta the property $\left(\mathrm{b}^{\prime}\right)$ implies $\left(\mathrm{c}^{\prime}\right)$; and $\left(\mathrm{a}^{\prime}\right)$ is equivalent to $\left(\mathrm{a}^{\prime \prime}\right): \operatorname{dim}_{\mathbb{Q}} X \leq 3$, provided $n \geq 3$.

Proof. Show that $\left(\mathrm{b}^{\prime}\right)$ implies $X \tau S U_{(0)}$. According to Theorem 10 of [7] $X \tau S U_{(0)}$ is equivalent to $\operatorname{dim}_{\pi_{i}} X \leq i$ where $\pi_{i}=\pi_{i}\left(S U_{(0)}\right)$ is a vector space over $\mathbb{Q}$. So, since only the third (and higher) homotopy group of $S U_{(0)}$ is not trivial, $X \tau S U_{(0)}$ is equivalent to $\operatorname{dim}_{\mathbb{Q}} X \leq 3$ and $\left(\mathrm{b}^{\prime}\right)$ implies that.

According to Bott's theorem $\pi_{i}\left(S O_{(0)}\right)=\mathbb{Q}$ if $i=8 k+3$ or $i=8 k-1$ and $=0$ otherwise. Then again by Theorem 10 of [7] $\left(a^{\prime \prime}\right)$ implies $\left(a^{\prime}\right)$. In order to complete the proof we need a relative version of Theorem 6 of [7].

We recall that $S P^{n} Y$ denotes the $n$-th symmetric power of the space $Y$. For a pointed space $Y$ there is a natural imbedding $S P^{n} Y \rightarrow S P^{n+1} Y$ for any 
$n$. We define the infinite symmetric power of $Y$ as the direct limit $S P^{\infty}=$ $\lim S P^{n} Y$.

Theorem 5. For any compact metric space $X$, every $A N R$-pair $(M, N)$, and for every $i=1,2, \ldots, \infty$, the property $X \tau(M, N)$ implies $X \tau\left(S P^{i} M, S P^{i} N\right)$.

Proof. The proof of the theorem does not differ much from that of Theorem 6 of [7]. So we omit it and note that with a slight modification the proof works for non-compact metric spaces.

Completion of the proof of Lemma 4. Apply Theorem 5 to obtain

$$
X \tau\left(S P^{\infty}\left(S O_{(0)}\right), S P^{\infty}\left(S O(n)_{(0)}\right)\right) .
$$

According to a theorem of Dold-Thom [8], [9] for every ANR-space $M$ we have a homotopy equivalence $S P^{\infty} M \sim \lim _{m} \prod_{i=1}^{m} K\left(H_{i}(M), i\right)$, where $H_{i}(M)$ means the $i$-dimensional integer homology group of $M$. The integer homology groups of $S_{(0)}$ coincide with the rational homology groups of $S O$ which are equal to rational homologies of the product $S^{3} \times S^{5} \times S^{7} \times \cdots$. For $n \geq 3$ the same is true only with the finite product for homologies of $S O(n)_{(0)}$. Note that in dimension 3 the inclusion $S O(n)_{(0)} \rightarrow S O_{(0)}$ induces an isomorphism in homologies $(n \geq 3)$.

So, there are homotopy equivalences $h_{1}: K(\mathbb{Q}, 3) \times N_{1} \rightarrow S P^{\infty}(S O(n))_{(0)}$ and $h_{2}: S P^{\infty}\left(S O_{(0)}\right) \rightarrow K(\mathbb{Q}, 3) \times M_{1}$, where $M_{1}$ and $N_{1}$ are 3-connected spaces, and the composition $h=h_{2} \circ i_{n} \circ h_{1}$ induces an isomorphism of 3dimensional homotopy groups. Hence the restriction of the composition $w \circ h$ of $h$ and the projection onto the first factor $w: K(\mathbb{Q}, 3) \times M_{1} \rightarrow K(\mathbb{Q}, 3)$ induces an isomorphism of 3-dimensional homotopy groups and therefore $h^{\prime}=$ $w \circ h$ is a homotopy equivalence.

Now let $f: A \rightarrow K(\mathbb{Q}, 3)=K(\mathbb{Q}, 3) \times\{p t\}$ be a partial map. By the assumption and Theorem 5 the map $i_{n} \circ h_{1} \circ f$ has an extension $\bar{f}: X \rightarrow S P^{\infty}\left(S O_{(0)}\right)$. Note that $w \circ \bar{f}$ restricted on $A$ coincides with $h^{\prime} \circ f$. Denote by $g^{\prime}$ a homotopy inverse map to $h^{\prime}$. We know that the map $h^{\prime} \circ f$ is extendable. Then the map $g^{\prime} \circ h^{\prime} \circ f$ is extendable. Since $g^{\prime} \circ h^{\prime}$ is homotopic to the identity map, the composition $g^{\prime} \circ h^{\prime} \circ f$ is homotopic to $f$. The Homotopy Extension Theorem implies that there exists an extension of $f$. Since $f$ is an arbitrary partial map, we have $\operatorname{dim}_{\mathbb{Q}} X \leq 3$.

We summarize the above in the following theorems.

Theorem 6. Let $X$ be a compact metric space. Then the following are equivalent:

(1) $M_{n}\left(C_{\mathbb{R}}(X)\right)$ has the weak power substitution for some $n \geq 3$.

(2) $\operatorname{dim}_{\mathbb{Q}} X \leq 3$.

(3) $M_{n}\left(C_{\mathbb{R}}(X)\right)$ has the weak power substitution for all $n$.

Note that $M_{n}\left(C_{\mathbb{R}}(X)\right)$ always has the power substitution for $n=1,2$ [2], [3].

Theorem 7. Let $X$ be a compact metric space. Then the following are equivalent:

(1) $M_{n}\left(C_{\mathbb{C}}(X)\right)$ has the weak power substitution for some $n$.

(2) $\operatorname{dim}_{\mathbb{Q}} X \leq 1$.

(3) $M_{n}\left(C_{\mathbb{C}}(\bar{X})\right)$ has the weak power substitution for all $n$. 


\section{3. n-POWER SUBSTITUTION}

Let $(-)^{n}: S^{1} \rightarrow S^{1}$ be a map of the circle to itself of degree $n$ (taking the $n$ th power). It follows by [3] that the ring of complex functions on a compactum $X$ has $n$-power substitution if and only if $X \tau(-)^{n}$.

Theorem 8. For every $n$ and every prime $p$ there exist an $n$-dimensional compactum $X$ with the $p^{n-1}$-power substitution of $C_{\mathbb{C}}(X)$.

Corollary 9. There exist a compactum $X$ such that the space of units $U C_{\mathbb{C}}(X)$ is not dense in $C_{\mathbb{C}}(X)$ and $U C_{\mathbb{C}}(X)$ is dense in $C_{\mathbb{C}}(X)^{2}=\left\{f^{2}: f \in C_{\mathbb{C}}(X)\right\}$.

Proof. The compactum $X$ of the theorem for $n=p=2$ has the requisite properties. First, we recall that if $U C_{\mathbb{C}}(X)$ is dense in $C_{\mathbb{C}}(X)$, then $\operatorname{dim} X \leq 1$ [6]. Since $\operatorname{dim} X \geq 2$, the units are not dense in $C_{\mathbb{C}}(X)$. Note that $X$ has the property $X \tau(-)^{2}$.

Let $f: X \rightarrow \mathbb{C}$ and $\varepsilon>0$ be given; we construct $g: X \rightarrow \mathbb{C}-\{0\}$ to be $\varepsilon$-close to $f^{2}$. Let $S_{\varepsilon}^{1}=\{z \in \mathbb{C}:|z|=\varepsilon\}$ be the circle bounding the disk $D_{\varepsilon}$ in the complex plane $\mathbb{C}$. Denote $A=f^{-1}\left(S_{\varepsilon}^{1}\right)$ and $Y=f^{-1}\left(D_{\varepsilon}\right)$. According to the property $X \tau(-)^{2}$ there is an extension of $\left.(-)^{2} \circ f\right|_{A}: A \rightarrow S_{\varepsilon^{2}}^{1}$ over $Y$ to a map $p: Y \rightarrow S_{\varepsilon^{2}}^{1}$. Glue maps $\left.f^{2}\right|_{X-Y}$ and $p$ to obtain a map $p^{\prime}: X \rightarrow \mathbb{C}-\{0\}$ which is $\varepsilon$-close to $f^{2}$.

Let $(X, Y)$ be a pair of spaces, $Y \subset X$, and let $g: L \rightarrow M$ be a map. Denote by $(X, Y) \tau g$ the property that for every map $f: Y \rightarrow L$ there is a map $q: X \rightarrow M$ such that $q$ is an extension of the composition $g \circ f$.

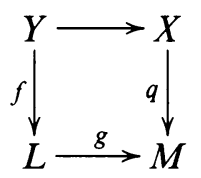

For every $h: Z \rightarrow X$ we denote the pair $\left(h^{-1}(X), h^{-1}(Y)\right)$ by $h^{-1}(X, Y)$.

We need the following lemmata.

Lemma 10. For any simplicial complex $(L, \pi)$ over the $n$-dimensional simplex $\sigma^{n}$ and for every prime $p$ there is a map of an n-dimensional polyhedron $\xi: R \rightarrow$ $L$ such that:

(1) for every simplex $\Delta \subset L$ the property $\xi^{-1}(\Delta, \partial \Delta) \tau(-)^{p}$ holds, where $(-)^{p}: S^{1} \rightarrow S^{1}$ denotes taking the $p$-th power on the unit circle,

(2) $\xi: H^{n}\left(L, \mathbb{Z}_{p}\right) \rightarrow H^{n}\left(R ; \mathbb{Z}_{p}\right)$ is an isomorphism.

By the definition in [11] a simplicial complex over the simplex $\sigma$ is a polyhedron $L$ with a simplicial map $\pi: L \rightarrow \sigma$ such that the restriction of $\pi$ on every simplex of $L$ is injective. Note that the first barycentric subdivision of any triangulation of the $n$-dimensional polyhedron $L$ defines a structure of simplicial complex over the $n$-simplex.

Proof. A similar lemma is proved in [5].

We apply induction on $n$. For $n=1$ we may define $R=L$ and $\xi=$ id.

In order to make the inductive step from $n$ to $(n+1)$ we need to construct that resolution for the $(n+1)$-dimensional simplex $\sigma$. Then for an arbitrary simplicial complex $(L, \pi)$ over the $(n+1)$-simplex we may apply a pull-back construction. 
Assume that $\xi_{Y}: Y \rightarrow \partial \sigma$ is such a resolution for the boundary of the $(n+1)-$ simplex with some triangulation turning it into a complex over the $n$-simplex. Let $\alpha_{1}, \ldots, \alpha_{m}$ be a basis of the 1-dimensional cohomology group $H^{1}(Y)$. By $\phi_{i}$ we denote a map $\phi_{i}: Y \rightarrow S^{1}$ which generates the element $p \alpha_{i}$. Let $T^{m}=S^{1} \times \cdots \times S^{1}$ be an $m$-dimensional torus and let $T_{n}^{m}$ denote the $n$ skeleton of $T^{m}$ with respect to the natural cellular structure. The diagonal product $\Delta \phi_{i}: Y \rightarrow T^{m}$ has a cellular approximation $\phi: Y \rightarrow T_{n}^{m}$. Let $M_{\phi}$ be a mapping cylinder, and define a map $\xi: M_{\phi} \rightarrow \sigma$ as the natural linear extension of $\xi_{Y}$.

First note that the property (1) holds. To check the property (2) we consider the diagram:

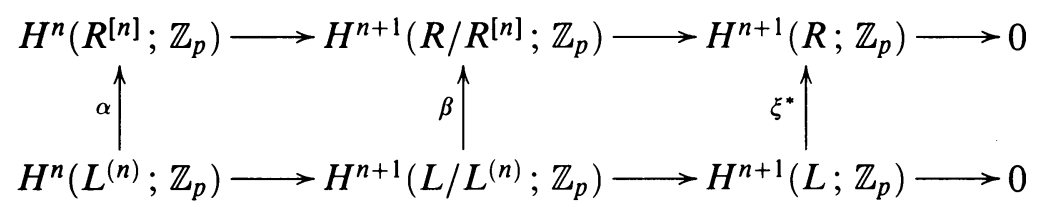

Here $L^{(n)}$ denotes the $n$-dimensional skeleton of a simplicial complex $L$ over the $(n+1)$-simplex $\sigma$ and $R^{[n]}$ denotes its preimage under $\xi$. Note that $\alpha$ is an isomorphism by the induction assumption. The quotient space $R / R^{[n]}$ is homeomorphic to the wedge of cones $\bigvee_{\sigma} C_{\phi}$ the map $\phi$. Hence, to complete the proof by applying the five-lemma it is sufficient to show that the projection $\gamma: C_{\phi} \rightarrow S^{n+1}$ induces an isomorphism of cohomology groups in dimension $n+1$. Since the map $\Delta \phi_{i}$ induces a zero homomorphism $\bmod p$ in dimension 1 , due to the ring structure on $H^{*}\left(T^{m}\right)$ it induces a zero homomorphism for $n$-dimensional cohomology $\bmod p$. Whence $\phi^{*}: H^{n}\left(T_{n}^{m} ; \mathbb{Z}_{p}\right) \rightarrow H^{n}\left(Y ; \mathbb{Z}_{p}\right)$ is a zero homomorphism. The Puppe exact sequence gives a diagram:

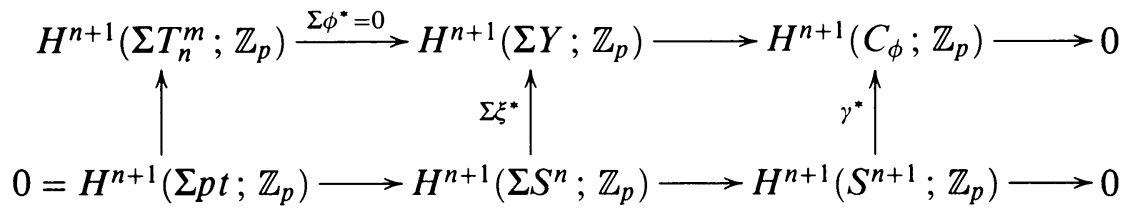

Since $\xi_{Y}^{*}$ is an isomorphism in the dimension $n, \gamma^{*}$ is also an isomorphism.

Lemma 11. Let a compactum $X=\lim \left\{P_{i} ; q_{i}^{i+1}\right\}$ be a limit space of the inverse system of $n$-dimensional polyhedra provided with triangulations $\tau_{i}$ such that

(1) $\lim \operatorname{mesh} q_{k}^{k+i}\left(\tau_{k+i}\right)=0 \forall k$,

(2) $\left(q_{i}^{i+1}\right)^{-1}(L)$ is a subpolyhedron of $P_{i+1}$ with respect to $\tau_{i+1}$ for every subcomplex $L \subset P_{i}$,

(3) $\left(\left(q_{i}^{i+1}\right)^{-1}(\sigma, \partial \sigma)\right) \tau(-)^{p}$ holds for every simplex $\sigma \in \tau_{i}$.

Then $X \tau(-)^{p^{n-1}}$.

Proof. Let $A$ be a closed subset of $X$ and $\phi: A \rightarrow S^{1}$ be an arbitrary map. Since $S^{1}$ is an ANR, for large enough $i$ there is a subcomplex $A_{i} \subset P_{i}$ with respect to the triangulation $\tau_{i}$ and a map $\phi_{i}: A_{i} \rightarrow S^{1}$ such that the restriction $\left.\phi_{i} \circ q_{i}^{\infty}\right|_{A}$ is homotopic to $\phi$. Since $S^{1}$ is path-connected, there is an extension $\phi^{\prime}: P^{(1)} \cup A_{i} \rightarrow S^{1}$ of $\phi_{i}$. Let $\psi_{1}=\phi^{\prime} \circ q_{i}^{i+1}$ and $W_{n}=\left(q_{i}^{i+1}\right)^{-1}\left(P_{i}^{(n)} \cup A_{i}\right)$. Apply the condition (3) to all 2-dimensional simplexes $\sigma$ to obtain a map 
$\psi_{2}: W_{2} \rightarrow S^{1}$ such that $(-)^{p} \circ \psi_{1}=\left.\psi_{2}\right|_{W_{1}}$. Then apply the condition (3) to all 3-simplexes to obtain a map $\psi_{3}: W_{3} \rightarrow S^{1}$ such that $(-)^{p} \circ \psi_{2}=\left.\psi_{3}\right|_{W_{2}}$ and so on. Then the map $(-)^{p^{n-1}} \psi_{1}=\psi_{n}$. This means that the composition $(-)^{p^{n-1}} \circ \phi_{i}$ has an extension over $P_{i+1}$. Hence $\left.(-)^{p^{n-1}} \circ \phi_{i} \circ q_{i}^{\infty}\right|_{A}$ has an extension and by the Homotopy Extension Theorem the map $(-)^{p^{n-1}} \circ \phi$ has an extension.

Proof of Theorem 8. By induction we construct an inverse system $\left\{P_{i} ; q_{i}^{i+1}\right\}$ with the properties (1)-(3) of Lemma 11 such that $q_{i}^{i+1}$ induces an isomorphism of the $n$-dimensional cohomology group with $\mathbb{Z}_{p}$ coefficients for every $i$. We take $P_{1}=S^{n}$ and consider an arbitrary triangulation $\tau_{1}$ with mesh $<1$ which turns $P_{1}$ into a simplicial complex over the $n$-simplex. Then we apply Lemma 10 to construct $q_{1}^{2}: P_{2} \rightarrow P_{1}$ and define a triangulation $\tau_{2}$ on $P_{2}$ such that $\operatorname{mesh}\left(\tau_{2}\right), \operatorname{mesh}\left(q_{1}^{2}\left(\tau_{2}\right)\right)<1 / 2$ and so on. According to Lemma $11 X$ has the property $X \tau(-)^{p^{n-1}}$. The dimension of $X \leq n$ since all $P_{i}$ are $n$-dimensional. Since $q_{1}^{\infty}$ induces an isomorphism of the $n$-dimensional $\bmod p$ cohomology groups, $X$ has $n$-dimensional $\bmod p$ cohomology non-trivial and hence is at least $n$-dimensional. Thus $\operatorname{dim} X=n$.

\section{BIBLIOGRAPHY}

1. K. R. Goodearl, Power-cancellation of groups and modules, Pacific J. Math. 64 (1976), 387-411.

2. __ Cancellation of low-rank vector bundles, Pacific J. Math. 113 (1984), 289-302.

3. R. Camps and P. Menal, The power substitution property for rings of continuous functions, preprint, 1993.

4. V. I. Kuzminov, Homological dimension theory, Russian Math. Surveys 23 (1968), 1-45.

5. A. N. Dranishnikov, Homological dimension theory, Russian Math. Surveys 43 (1988), no. 4, 11-63.

6. L. N. Vaserstein, Stable rank of rings and dimensionality of topological spaces, Funct. Anal. Appl. 5 (1971), 102-110.

7. A. N. Dranishnikov, An extension of mappings into $C W$-complexes, Math. USSR-Sb. 74 (1993), no. 1, 47-56.

8. A. Dold and R. Thom, Quasifaserungen und unedliche symmetrische Produkte, Ann. of Math. (2) 67 (1958), 239-281.

9. P. Hilton, Homotopy theory and duality, Gordon and Breach, New York, 1965.

10. D. Sullivan, Geometric topology, Part I: Localization, periodicity, and Galois symmetry, M.I.T. Press, Cambridge, MA, 1970.

11. R. F. Williams, $A$ useful functor and three famous examples in topology, Trans. Amer. Math. Soc. 106 (1963), 319-329.

Department of Mathematics, Cornell University, Ithaca, New York 14853-7901

Current address: Department of Mathematics \& Computer Science, University of Miami, Coral Gables, Florida 33124

E-mail address: dranish@paris.miami.edu 